\title{
Development of an Air Volume Measuring Instrument: WINSPEC
}

\author{
Kouetsu Tanaka, Shintaro Sakamoto, Yasunori Abe \\ Research and Development Center \\ Shinryo Corporation \\ E-mail:tanaka.ko@shinryo.com
}

\begin{abstract}
Prior to completion, the various facilities associated with a building undergo performance testing. For air conditioning facilities, it is necessary to confirm that each apparatus meets the design values for air supply and emission volume. In keeping, rather than a fully automated robot, we developed a human-operated instrument that enables ceiling outlets to be reached without using a stepladder; we also engaged in development to make it possible for the instrument to move smoothly around worksites. This instrument has been used in actual completion inspections, and results have been obtained for dozens of properties to the present. Although WINSPEC has primarily contributed to lowering costs through reduced labor power and shortened work time, it is also used for the purpose of guaranteeing measurement accuracy. This report provides an overview of WINSPEC and presents how the instrument has been evaluated from results of actual use at construction sites.
\end{abstract}

Keywords: air volume, anemometer, ceiling, human-operated instrument

\section{INTRODUCTION}

Air-conditioning systems in buildings are designed to provide a pleasant environment for both people and machinery by controlling the temperature, humidity, flow rate and cleanliness (including $\mathrm{CO}_{2}$ concentration) of the air supply. Air-conditioning systems also require measurement and analysis to ascertain that the air supply meets the design requirements.

Air volume is one of the required measurements, used to ascertain whether the air volume from the outlets is consistent with the original design requirements. Air volumes are normally measured at all outlet vents.

Large buildings can have thousands of outlet vents, if not tens of thousands. In a bid to facilitate the task of inspection of large numbers of vents in the busy period prior to completion of a building, we developed an

independently-propelle

d robot capable of automatically

identifying

and

measuring air volume at

vents throughout the building $^{[1]-[3]}$ (see Fig.1). However, implementing the robot in an actual on-site situation proved difficult for two main reasons. Firstly, site conditions are constantly changing and evolving on account of continual modifications and alterations being made on a daily basis, and this necessitates the use of highly advanced technology such as collision avoidance systems. Also, with so many different contractors working in the same area at the same time, safety is an important consideration. In practice, it is virtually impossible to create an on-site environment in which a robot can move from place to place without hindrance.

Nevertheless, the enormous labor-saving potential of this approach convinced us to switch our focus to developing an operator-guided inspection system.

The result was WINSPEC, a compact device designed for efficient and accurate air volume measurement. The first WINSPEC prototype was completed in 2000, and subsequently underwent a number of design revisions and modifications, including development of a second model specifically for production plants. To date, WINSPEC has been used on over 50 construction sites.

This paper describes the basic design and systems technology employed in WINSPEC, and analyses the performance of the machine in actual on-site conditions.

\section{SPECIFICATIONS}

JIS-A1431 describes the official requirements pertaining to measurement of air volume from air-conditioning equipment. However, the JIS approach can be costly and labor-intensive because its system is too large. For this reason, many construction sites use alternative methods that are simpler but equally accurate. Several examples are given below.

The majority of such methods involve calculating the air volume by measuring the air velocity at the vent (by means of an operator standing on a ladder holding a portable anemometer close to the ceiling) and multiplying this value by the surface area of the vent. Where the vent is fitted with diffuser panels, it must be covered over with a straight duct large enough to enclose the entire surface area. The air 
velocity is measured at several points inside the duct and the measurements are averaged out. The average value is then multiplied by the cross-sectional area of the duct to determine the air volume.

The main problems with this approach are as follows.

(1) Poor accuracy - a minor difference in position can significantly affect the air flow speed

(2) Measurement accuracy is easily affected by operator and measurement method

(3) Carrying around a ladder is both tiring and inefficient

(4) Labor-intensive - all measurement data must be matched to vent locations

In light of these issues, we decided to design a fully-equipped cart-style machine that could be guided by hand through the unique environment of the construction site and that could also extend upwards to ceiling vents. The basic operating requirements of the machine were as follows:

\section{(1) Accurate air volume measurement}

(2) Two separate models: one for standard office buildings with ceiling height of $3 \mathrm{~m}$ and the other for production plants with ceiling height of $4.5 \mathrm{~m}$

(3) Lightweight and easily transported around the site area

(4) Battery-operated and cable-free

(5) Equipped with storage media to hold measurement data for subsequent report writing

The next section describes the WINSPEC range of machines designed to meet the above requirements.

\section{WINSPEC OVERVIEW}

There are three WINSPEC models designed for different operating environments, as shown in Fig.2.

\section{(1) WINSPEC-2}

The standard WINSPEC model designed for ordinary office buildings. It is lightweight and compact and can be used with ceiling heights of up to 3 meters.

\section{(2) WINSPEC-i}

Designed for production plants and other facilities with ceiling heights of up to 4.5 meters.

\section{(3) WINSPEC-P}

Designed for buildings that are already fitted out and other situations that are inaccessible to the standard WINSPEC model.
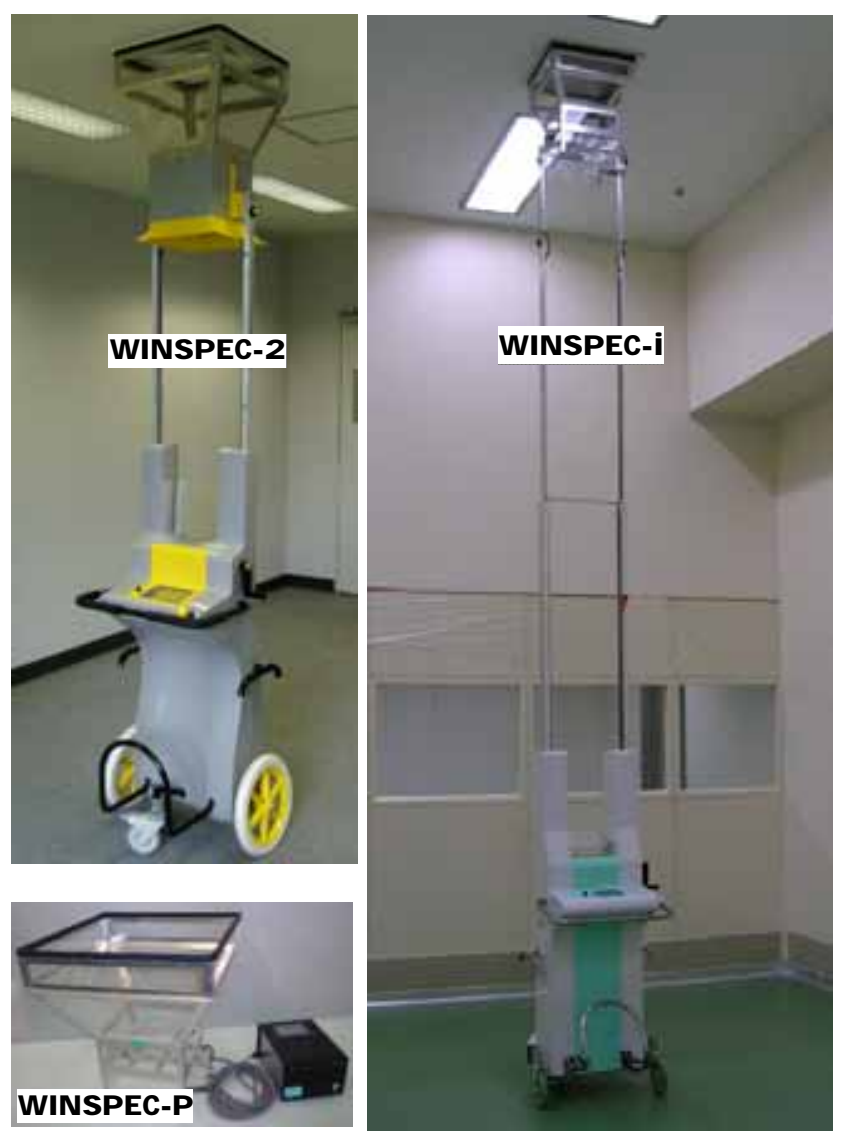

Fig.2 Appearance of the WINSPEC

The WINSPEC machine is broadly divided into three parts.

\section{(1) Air volume measurement}

The air volume measurement section consists of a sensor hood fitted with multiple air velocity sensors, a sensor board including constant-current and bridged circuits, and an interchangeable hopper attachment (attachment hood) that can be changed to suit the size of the air vent.

\section{(2) Operation}

The device is operated via a user-friendly touch-panel screen. A microcomputer processes the touch-panel commands and the output from the air velocity sensors. Observation data can be written to storage media such as floppy disks or compact flash cards.

\section{(3) Cart}

The cart base is equipped with a riser mechanism for projecting the attachment hood and sensor hood up to the ceiling. The riser is operated manually in order to minimize the overall weight of the WINSPEC. The microcomputer and other electronic components are powered by a battery and charging unit. The cart base is fitted with wheels so that it can be transported around the construction site with ease. WINSPEC-2 and WINSPEC-i are also equipped with plastic or aluminum covers. 


\subsection{Air volume measurement}

\subsubsection{Measurement method}

The method prescribed in JIS involves fitting a hood to the air vent and measuring the air velocity at measurement points in a straight duct. However, an auxiliary suction fan must also be used to eliminate pressure losses in the measurement section, thereby ensuring that the static pressure in the hood is the same as the ambient static pressure. As mentioned above, when it comes to large systems, this approach is impractical with regards to both the measurement process and transportation of the equipment.

The WINSPEC calculates the air volume by taking simultaneous measurements of air velocity at multiple points in a channel fitted with air velocity sensors.

The attachment hood is matched to the size of the air vent and used to collect the air into the sensor hood, where air velocity measurement takes place. There are three types of sensor hood: cross-sectional area of 250 x $250 \mathrm{~mm}$ fitted with nine air velocity sensors; $300 \times 300 \mathrm{~mm}$ with nine sensors; and $300 \times 600 \mathrm{~mm}$ with 15 sensors. The choice of sensor hood is determined by the air velocity, with the aim being to achieve a through flow in the range $1-10 \mathrm{~m} / \mathrm{sec}$.

Although the attachment hood does generate a certain degree of pressure loss, considering the weight of the equipment that would be necessary to eliminate this loss, plus the increased battery weight, it was considered unfeasible to deal with. The pressure loss has the effect of making the air volume figures slightly lower than the "real" values. This is considered acceptable since it errs on the side of safety.

\subsubsection{Air velocity sensors}

The air velocity sensor probes are ø $2.5 \mathrm{~mm}$ spherical aluminum bulbs containing embedded germanium chips (see Fig.3), which are heated via a constant current of 30 $\mathrm{mA}$. The surface temperature of the probe reaches around $150^{\circ} \mathrm{C}$. As the temperature of the germanium rises, its electrical resistance decreases and the voltage also drops. When the probe comes into contact with air at a temperature lower than the probe surface temperature, the germanium is cooled, the electrical resistance increases and the voltage rises. A higher air velocity causes greater heat loss in the sensor, reducing its temperature and increasing the voltage.

The relationship between air velocity and voltage is determined in calibration tests using a standard air velocity meter. When this relationship is known, the probes

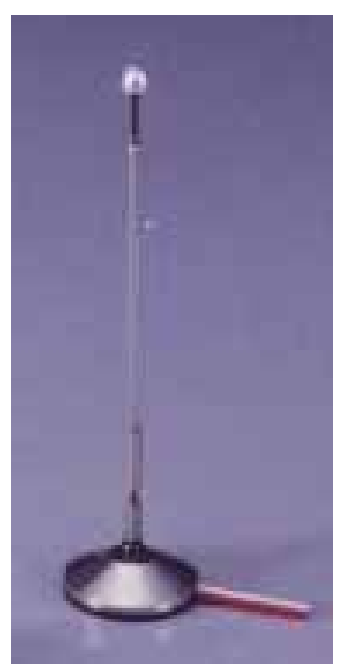

Fig.3 Air Velocity Sensor Probe effectively function as air velocity sensors.

Air temperature sensors operate on much the same principle: a constant current of $50 \mathrm{~mA}$ is applied to a silicon thermistor, and voltage fluctuations indicate change in temperature. Air temperature sensors are used for temperature correction of the readings from the air velocity sensors. The sensor probes are omni-directional and are equally suitable for air intake measurement.

\subsubsection{Sensor board}

The WINSPEC sensor board is connected to five air velocity sensor probes and one air temperature sensor probe. It supplies a constant current to the probes, and performs $\mathrm{AD}$ conversion on the sensor output voltages while switching between them with a multiplexer. Serial communication is controlled by a microcomputer.

\subsection{Operation and controls}

Overall operation of the system is controlled by an 8-bit microcomputer. Fig. 4 shows the configuration of the other electronic components.

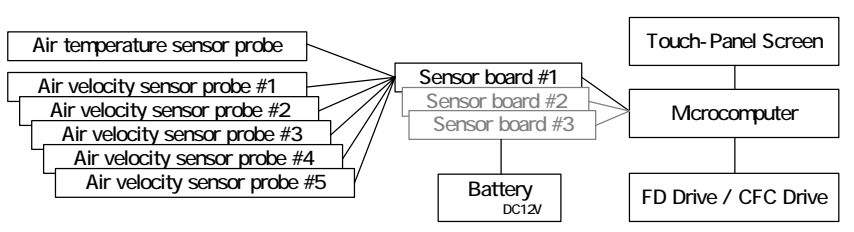

Fig.4 System block

The touch-panel screen functions as a combined display and operation panel. A user-friendly dialog-style interface has been employed to ensure that the device is easy to use.

When the Start Measurement button on the screen is pressed, measurement begins after a delay of approximately eight seconds. Air velocity sensor data is sampled ten times at intervals of one second. The average of these values is then multiplied by the cross-sectional area of the sensor hood to produce the air volume, which is displayed on the screen.

Other display functions include measurement history, on-screen correction of air volume, low battery warning, and sensor malfunction warning.

Observation air velocity data is recorded together with the air vent number, room name, air temperature and calculated air volume. Data is stored in CSV format on floppy disk or compact flash card media for transfer to computer.

\subsection{Cart}

\subsubsection{Vertical lifting mechanism}

The vertical lifting mechanism is a manually operated device used to raise the attachment hood up to the ceiling. The rotary ratchet handle is used to raise the measurement section of the WINSPEC to the required height, then the 

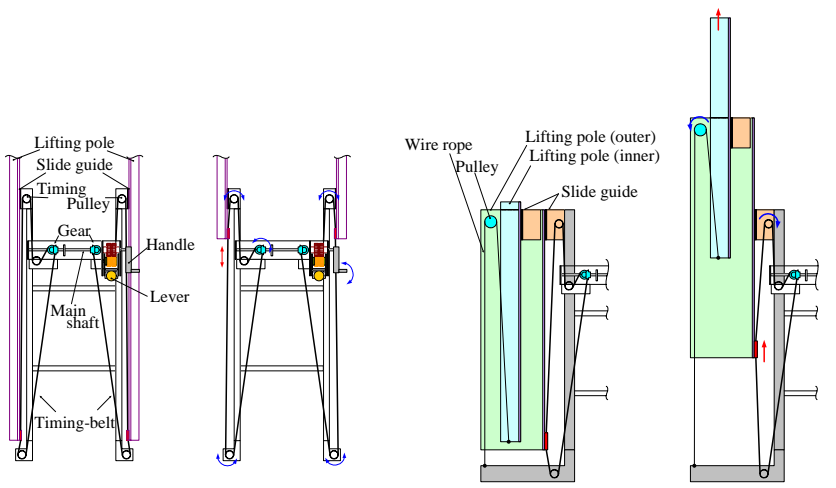

Fig.5 Vertical lifting mechanism

stopper holds it in position. As Fig.5 shows, the WINSPEC-i has a two-stage sliding mechanism 1.5 meters in length and capable of extending to 2.4 meters to reach ceilings up to 4.5 meters in height.

The mechanism is lowered by pressing the lever to remove the stopper, whereupon the attachment and sensor hoods descend from the ceiling under their own weight at a gentle speed regulated by rotary dampers.

\subsubsection{Power supply}

Electronic components are powered by the DC $12 \mathrm{~V}$ battery. The battery holds enough charge for five hours of continuous usage with a system configured with 15 sensors. The number of sensors governs the battery consumption and therefore maximum usage time. The battery is recharged via the built-in recharger.

\subsubsection{Transport}

The WINSPEC machine is pushed forward using the handling bar. WINSPEC-2 has two main wheels, one on each side, plus two casters, at the front and the rear. WINSPEC-i has three casters, one on each side and one at the front, and two casters at each side which extend during measurement to provide extra stability while the sensors are in the elevated position (see Fig.6). The casters fold back when not required to allow passage through doorways and into elevators.

\subsection{Specifications}

Table 1 shows the main specifications of the WINSPEC-i and the WINSPEC-2.
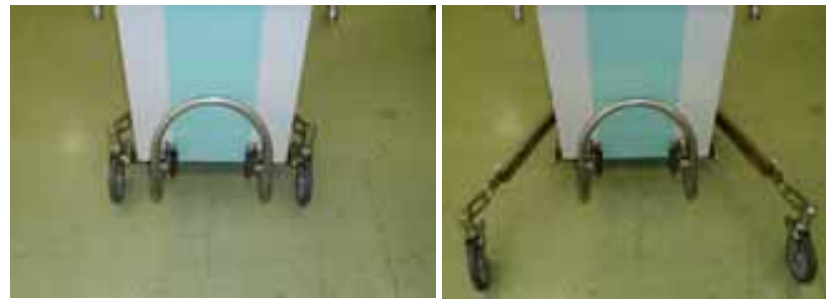

Fig.6 Outrigger type caster

\section{PERFORMANCE EVALUATION}

\subsection{Basic performance criteria}

\subsubsection{Measurement accuracy}

This section describes the measurement accuracy of the WINSPEC machine. For the purpose of this section, the air velocity sensor probes, sensor board and microcomputer are collectively referred to as WINSPEC.

WINSPEC is required to deliver accuracy equivalent to that of a standard air velocity meter as defined in JIS-T8202. JIS stipulates an accuracy of within $\pm(5 \%+0.1)$ $\mathrm{m} / \mathrm{s}$ of the reading value, although at the lowest air velocity this increases to $\pm 100 \%$. WINSPEC has been calibrated to an accuracy of within $\pm(5 \%+0.1) \mathrm{m} / \mathrm{s}$ of the reading value (see below) and therefore achieves the required level of accuracy.

Other parameters such as consistency of observations, the impact of fluctuations in air current temperature and response time have been demonstrated through experiment to fulfill the respective requirements.

Thus, the WINSPEC air velocity measuring system is considered to be the equivalent of a standard air velocity meter with respect to the JIS-T8202 requirements.

\subsubsection{Calibration method}

The WINSPEC air velocity sensors are calibrated in our own Gettingen type wind tunnel by comparison with a standard air velocity meter (a 3-D ultrasonic meter traceable to national standards). The WINSPEC measuring apparatus is placed next to the standard air velocity meter at the measuring point inside the tunnel. Calibration specifications are shown below.

(1) Wind tunnel

Type: Circular

Calibration range: $0.1-12.0 \mathrm{~m} / \mathrm{s}$

Calibration accuracy: within $\pm 1 \%$ of standard air velocity meter

Variable air velocity range: $0.05-12.0 \mathrm{~m} / \mathrm{s}$

Air velocity: set manually using potentiometer

Air velocity distribution: maximum \pm 1

Temperature range: $0-55^{\circ} \mathrm{C}$

Humidity range: $20 \%$ - 90\% RH

(2) Standard air velocity meter

Name: 3-D ultrasonic air velocity meter

Specifications

Method: time division transmit/receive switching ultrasonic pulse measurement

Calculation: ultrasonic propagation time reciprocal difference method

Measurement range: within 1\%

Resolution: within $0.005 \mathrm{~m} / \mathrm{s}$

Speed: 20 repetitions per second

(3) Standard temperature gauge

Type: Precision temperature gauge No. 1 
Measurement range: $0-50^{\circ} \mathrm{C}$

Scale: $0.1^{\circ} \mathrm{C}$ increments

\subsubsection{Traceability}

(1) Standard air velocity meter

The 3-D ultrasonic air velocity meter is calibrated on an annual basis by the Japan Quality Assurance Organization (JQA). Calibration is performed using air velocity standard values traceable to national standards issued by the National Institute of Advanced Industrial Science and Technology (AIST).

\section{(2) Standard temperature gauge}

The standard temperature gauge is calibrated on an annual basis by the temperature gauge manufacturer Shin-Nippon Keisoku Co., Ltd. Calibration is performed using a standard glass temperature gauge traceable to national standards from the National Institute of Advanced Industrial Science and Technology.

\subsection{Practical performance}

The WINSPEC system performed admirably in tests conducted in actual on-site conditions.

In ordinary office buildings, WINSPEC achieved excellent results on system line ceilings featuring a connected series of identical vents. In production plants, meanwhile, WINSPEC was well received since it was capable of reaching high ceilings without need for a rolling tower, and proved an efficient means of acquiring the required measurements from a large number of vents. For example, in a factory with 4.5-meter ceilings and large vents measuring $1.5 \mathrm{~m}$ x $750 \mathrm{~mm}$, a single WINSPEC machine was able to measure 200 vents in a day, thus achieving substantial time and money savings.

\section{CONCLUSIONS}

This report supports the following conclusions regarding the newly developed WINSPEC machine:

(1) WINSPEC is suitable for use by a single operator and does not require a stepladder; and

(2) WINSPEC is calibrated and traceable to national standards, thus eliminating potential human error and ensuring a high level of accuracy.

In addition to providing an efficient and accurate means of measurement, the WINSPEC allows companies to devise their own approach for obtaining the required observations.

In the future, we will continue to modify and refine the WINSPEC to make the device even easier to use, and will introduce validation designed to suit specific factory environments.

\section{REFERENCES}

[1] Toshio FUKUDA, et al, "Navigation System based on Ceiling Landmark Recognition for Autonomous Mobile Robot", Proceedings of the 19th Annual Conference of the IEEE Industrial Electronics Society, pp.1466-1471, 1993

[2] Y. Abe, et al, "Development of air conditioning equipment inspection robot with vision based navigation system", Proceedings of the 11th International Symposium on Automation and Robotics in Construction, pp.665-674, 1994

[3] Yasunori ABE, et al, "Vision based Navigation System of Air Conditioning Equipment Inspection Robot", Proceedings of the 13th International Symposium on Automation and Robotics in Construction, pp.981-990, 1996

Table 1 Specifications

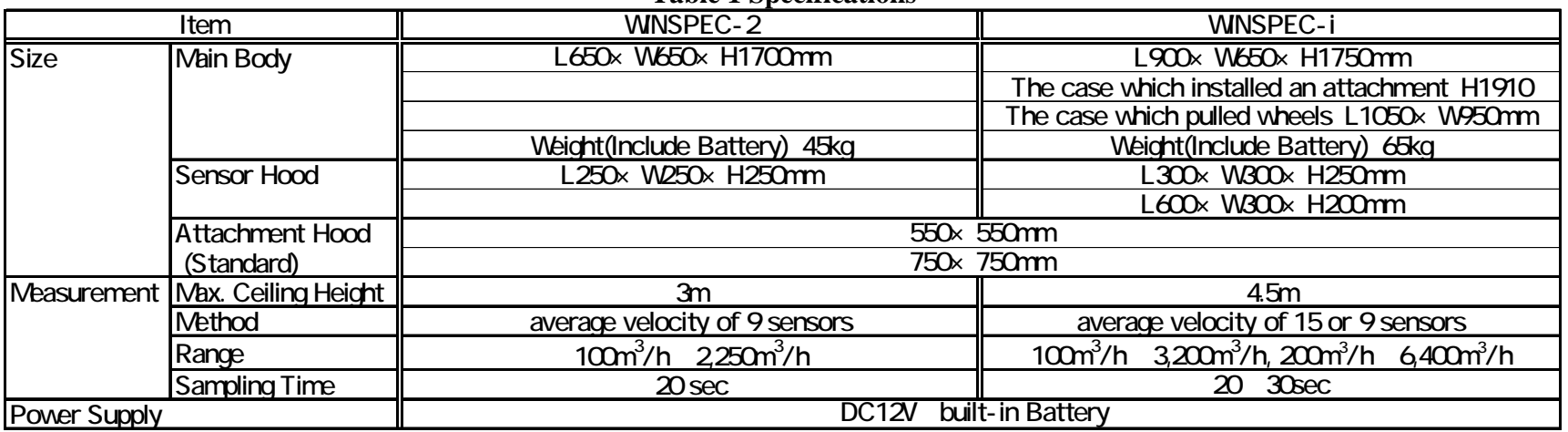

